\title{
Performance Comparison of MANET (Mobile Ad hoc Network) Protocols (ODMRP with AMRIS and MAODV)
}

\author{
Aparna K \\ Lecturer, Dept. of Master of Computer Applications \\ BMS Institute of Technology, Bangalore, India.
}

\begin{abstract}
In this paper, we present a comparative performance of three multicast protocols for Mobile Ad hoc Networks - ODMRP, AMRIS and MAODV focusing on the effects of changes such as the increasing number of receivers or sources and increasing the number of nodes. Although some simulation results of MANET protocols have been published before, these three protocols have not been compared in isolation. In recent years, a number of new multicast protocols have been proposed for ad hoc networks. A systematic performance evaluation of these protocols is done by performing certain simulations under NS-2. The applicability of multicast protocols to diverse situation are also studied and discussed.
\end{abstract}

\section{Keywords}

ODMRP, AMRIS, MAODV, MANETs, Multicasting

\section{INTRODUCTION}

A mobile ad hoc network (MANET) is a type of wireless networks. This type depends on the mobile nodes and there is no infrastructure in such type. There are no routers, servers, access points or cables. Nodes (mobiles) can move freely and in arbitrary ways, so it may change its location from time to time. Each node may be a sender or a receiver, and any node may work as a router and do all router functions. This means that it can forward packets to other nodes. Many applications of MANET's are implemented and used until today like in meeting conferences; military operations; search and rescue operations, all of them are examples of MANET networks.

Multicasting in wireless ad - hoc network is a hot topic in recent years. By multicasting, we mean the transmission of packets from a source or a group of sources to a group of one or more hosts that are identified by a single destination address. Multicasting greatly reduces the transmission cost when sending the same packet to multiple recipients.

It can improve the usage of wireless links by sending multiple copies of data packets using inherent broadcast behavior of wireless transmission though reducing transmission overhead and power consumption is a very challenging part in multicasting. There are many applications where one-to-many and many-tomany transmissions are required. The multicast service is employed in areas of collaborative work e.g. in rescue operations, battlefields video conferencing etc. Protocols used in static networks, for example, CBT, DVMRP, PIM do not perform well in dynamic environment.
The approach to do multicasting is basically classified into treebased and mesh-based approaches. A tree- based multicast routing protocol maintains either a single shared tree for all the transmissions or different trees from different sources to all the destinations of a multicast group. Tree-based routing protocols have only single path from source to destinations, so the broken links need to be repaired. On the other hand, mesh-based routing protocol maintains mesh of the connected components of the network and therefore, has multiple paths from sources to multicast destinations. This reduces repairing overhead due to presence of alternate paths available in the network. Mesh-based routing protocols lead to congestion under the conditions of high traffic load which can result in low packet delivery ratio. This paper summarizes the simulation techniques and analysis of some of the multicast protocols like MAODV [6] and ODMRP [3] and AMRIS in MANET environment.

The rest of the paper is organized as follows. A general description of MANET is depicted in section 2. The operation of the three protocols we studied ODMRP, MAODV and AMRIS is summarized in section 3 . The simulation environment is described in section 4 . We present results in section 5 and conclude with section 6 .

\section{GENERAL DESCRIPTION OF MANETs}

A MANET consists of mobile platforms (e.g., a router with multiple hosts and wireless communications devices) - herein simply referred to as "nodes" - which are free to move about arbitrarily. The nodes may be located in or on airplanes, ships, trucks, cars, perhaps even on people or very small devices, and there may be multiple hosts per router. A MANET is an autonomous system of mobile nodes. The system may operate in isolation, or may have gateways to and interface with a fixed network. In the latter operational mode, it is typically envisioned to operate as a "stub" network connecting to a fixed internetwork. Stub networks carry traffic originating at and/or destined for internal nodes, but do not permit exogenous traffic to "transit" through the stub network. MANET nodes are equipped with wireless transmitters and receivers using antennas which may be omni directional (broadcast), highly- directional (point-topoint), possibly steerable, or some combination thereof. At a given point in time, depending on the nodes' positions and their transmitter and receiver coverage patterns, transmission power levels and co-channel interference levels, a wireless connectivity in the form of a random, multihop graph or "ad hoc" network exists between the nodes. This ad hoc topology may change with 
time as the nodes move or adjust their transmission and reception parameters.

MANETs have several salient characteristics:

- Dynamic topologies: Nodes are free to move arbitrarily; thus, the network topology--which is typically multihop - may change randomly and rapidly at unpredictable times, and may consist of both bidirectional and unidirectional links.

- Bandwidth-constrained, variable capacity links: Wireless links will continue to have significantly lower capacity than their hardwired counterparts. In addition, the realized throughput of wireless communications - after accounting for the effects of multiple access, fading, noise, and interference conditions, etc., is often much less than a radio's maximum transmission rate.

- $\quad$ Energy-constrained operation: Some or all of the nodes in a MANET may rely on batteries or other exhaustible means for their energy. For these nodes, the most important system design criteria for optimization may be energy conservation.

- $\quad$ Limited physical security: Mobile wireless networks are generally more prone to physical security threats than are fixedcable nets. The increased possibility of eavesdropping, spoofing, and denial-of-service attacks should be carefully considered. Existing link security techniques are often applied within wireless networks to reduce security threats. As a benefit, the decentralized nature of network control in MANETs provides additional robustness against the single points of failure of more centralized approaches.

\section{Issues in Providing Multicast in MANET}

Well established routing protocols do exist to efficient multicasting service in conventional wired networks. These protocols, having been designed for fixed networks, may fail to keep up with node movements and frequent topology changes in a MANET. As nodes become increasingly mobile, these protocols need to evolve to provide efficient service in the new environment. Therefore, MANET, which completely lacks infrastructure, appears less promising. Host mobility increases the protocol overheads substantially. Rather, new protocols are being proposed and investigated that take issues such as topological changes into consideration. Moreover, the nodes of a MANET rely on batteries; thus routing protocols must limit the amount of control information passed between $n$ nodes.

The majority of applications are in areas where rapid deployment and dynamic reconfiguration are necessary and a wireline network is not available. These include military battlefields, emergency search and rescue sites, classrooms, and conventions where participants share information dynamically using their mobile devices. These applications lend themselves well to multicast operation. In addition, within a wireless medium, it is even more crucial to reduce transmission over-head and power consumption. Multicasting can improve the efficiency of the wireless links, when sending multiple copies of messages, by exploiting the inherent broadcast property of the wireless medium when multiple mobile nodes are located within the transmission range of a node. However, besides the issues for any ad hoc routing protocol listed above, wireless mobile multicasting faces several key challenges. Multicast group members can move, thus precluding the use of a fixed multicast topology. Transient loops may form during reconfiguration of distribution structure (e.g., tree) as a result of the mobility. Therefore, the reconfiguration scheme should be kept simple to maintain the channel overhead low. As we can see, providing efficient multicasting over MANET faces many challenges, including dynamic group membership and constant update of delivery path due to node movement.

\section{PROTOCOL DESCRIPTION}

\subsection{On-Demand Multicast Routing Protocol (ODMRP)}

ODMRP [1], [2], [3] creates a mesh of nodes (the "forwarding group") which forward multicast packets via flooding (within the mesh), thus providing path redundancy. ODMRP is an on-demand protocol, thus it does not maintain route information permanently. It uses a soft state approach in group maintenance. Member nodes are refreshed as needed and do not send explicit leave messages. In ODMRP, group membership and multicast routes are established and updated by the source on demand. Similar to ondemand unicast routing protocols, a request phase and a reply phase comprise the protocol. When multicast sources have data to send, but do not have routing or membership information, they flood a JOIN DATA packet. When a node receives a nonduplicate JOIN DATA, it stores the upstream node ID (i.e., backward learning) and rebroadcasts the packet. When the JOIN DATA packet reaches a multicast receiver, the receiver creates a JOIN TABLE and broadcasts to the neighbors. When a node receives a JOIN TABLE, it checks if the next node ID of one of the entries matches its own ID. If it does, the node realizes that it is on the path to the source and thus is part of the forwarding group. It then broadcasts its own JOIN TABLE built upon matched entries. The JOIN TABLE is thus propagated by each forwarding group member until it reaches the multicast source via the shortest path. This process constructs (or updates) the routes from sources to receivers and builds a mesh of nodes, the forwarding group. Multicast senders refresh the membership information and update the routes by sending JOIN DATA periodically. Another unique property of ODMRP is its unicast capability. Not only can ODMRP coexist with any unicast routing protocol, it can also operate very efficiently as unicast routing protocol. Thus, a network equipped with ODMRP does not require a separate unicast protocol.

\subsection{Ad hoc Multicast Routing protocol utilizing Increasing idnumberS (AMRIS)}

AMRIS is an on-demand protocol that constructs a shared multicast delivery tree to support multiple senders and receivers in a multicast session. AMRIS [4] establishes a shared tree for multicast data forwarding. Each node in the network is assigned a multicast session ID number. The ranking order of ID numbers is used to direct the flow of multicast data. Like ODMRP, AMRIS does not require a separate unicast routing protocol. Initially, a special node called Sid broadcasts a NEW-SESSION packet. The NEW-SESSION includes the Sid's msm-id (multicast session member id). Neighbor nodes, upon receiving the packet, calculate their own msm-ids which are larger than the one specified in the packet. The msm-ids thus increase as they radiate from the Sid. The nodes rebroadcast the NEW-SESSION message with the msm-id replaced by their own msm-ids. Each node is required to broadcast beacons to its neighbors. The beacon message contains the node id, msm-id, membership status, registered parent and child's ids and their msm-ids, and partition id. A node can join a multicast session by sending a JOIN-REQ. This JOIN-REQ is 
unicasted to a potential parent node with a smaller msm-id than the node's msm-id. The node receiving the JOIN-REQ sends back a JOIN-ACK if it already is a member of the multicast session. Otherwise, it sends a JOIN-REQ.PASSIVE to its potential parent. If a node fails to receive a JOIN-ACK or receives a JOIN-NAK after sending a JOIN-REQ, it performs "Branch Reconstruction (BR)." The BR process is executed in an expanding ring search until the node succeeds in joining the multicast session. AMRIS detects link disconnection by a beaconing mechanism. If no beacons are heard for a predefined interval of time, the node considers the neighbor to have moved out of radio range. If the former neighbor is a parent, the node must rejoin the tree by sending a JOIN-REQ to a new potential parent. If the node fails to join the session or no qualified neighbors exist, it performs the BR process. Data forwarding in done by the nodes in the tree. Only the packets from the registered parent or registered child are forwarded. Hence, if the tree link breaks, the packets are lost until the tree is reconfigured. Our AMRIS implementation followed the specification in [4].

BR consists of two subroutines. BR1 is executed when a node has a potential parent node for a group. If it does not find any potential parent node, BR2 is executed. In BR2, instead of sending a unicast JOIN-REQ to a potential parent node, the node broadcasts a JOIN-REQ that consists of a range field $\mathrm{R}$ to specify the nodes till $\mathrm{R}$ hops. Upon link breakage, the node with the larger msm-id tries to rejoin the tree by executing any of the BR mechanism. It is to be noted that AMRIS detects link disconnection by a beaconing mechanism. Hence, until the tree is reconstructed, packets could possibly be dropped.

\subsection{Multicast Ad Hoc On-demand Distance Vector (MAODV)}

MAODV routing protocol follows directly from unicast AODV, and discovers multicast routes on demand using a broadcast route discovery mechanism employing the same route request (RREQ) and route reply (RREP) messages that exist in the

send to a multicast group but does not have a route to that group. Only a member of the desired multicast group may respond to a join RREQ. If the RREQ is not a join request, it receives a RREQ and does not have a route to that group, it rebroadcasts the RREQ to its neighbors.

As the RREQ is broadcast across the network, nodes set up pointers to establish the reverse route in their route tables. A node receiving an RREQ first updates its route table to record the sequence number and the next hop information for the source node. This reverse route entry may later be used to relay a response back to the source. For join RREQs, an additional entry is added to the multicast route table and is not activated unless the route is selected to be part of the multicast tree. If a node receives a join RREQ for a multicast group, it may reply if it is a member of the multicast group's tree and its recorded sequence number for the multicast group is at least as great as that contained in the RREQ.

The responding node updates its route and multicast route tables by placing the requesting node's next hop information in the tables, and then unicasts an RREP back to the source. As nodes along the path to the source receive the RREP, they add both a route table and a multicast route table entry for the node from which they received the RREP, by creating the forward path. unicast AODV protocol. A mobile node originates an RREQ message when it wishes to join an multicast group, or has data to

\section{SIMULATION ENVIRONMENT}

The simulations of ODMRP, AMRIS and MAODV are implemented in ns2. Our simulation models a network of 50 mobile hosts placed randomly within a $1200 \mathrm{~m} \mathrm{X} \mathrm{1200m} \mathrm{area.}$ Radio propagation range is 250 meters in scenarios without unidirectional links. The channel capacity is $2 \mathrm{Mbit} / \mathrm{sec}$. There is a little temporal partition of the network. Each simulation executes for 400 seconds of simulation time. Multiple runs with different random seed number are conducted for each scenario and collected data is averaged over those runs. The multicast data streams are CBR streams with jitters. The size of data packet is 512 bytes. The multicast sources are selected from all 50 nodes randomly and most of them act as receivers at the same time. Receivers join one multicast group at the beginning of the simulation and never leave the group during the simulation. Nodes randomly select a destination and move with a predefined average speed. We have used the following metrics in comparing protocol performance.

- $\quad$ Packet Delivery Ratio: defined as the number of data packets delivered to multicast receivers over the number of data packets supposed to be delivered to multicast receivers. This ratio represents the multicasting effectiveness of the protocol. Higher value implies better performance.

- Multicast Efficiency: defined as the number of data packets delivered to multicast receivers over the number of total data packets forwarded. Higher value implies better performance. Multicast Efficiency= total received packets / total forwarded packets

\section{SIMULATION RESULTS}

\subsection{Effect of Number of Senders}

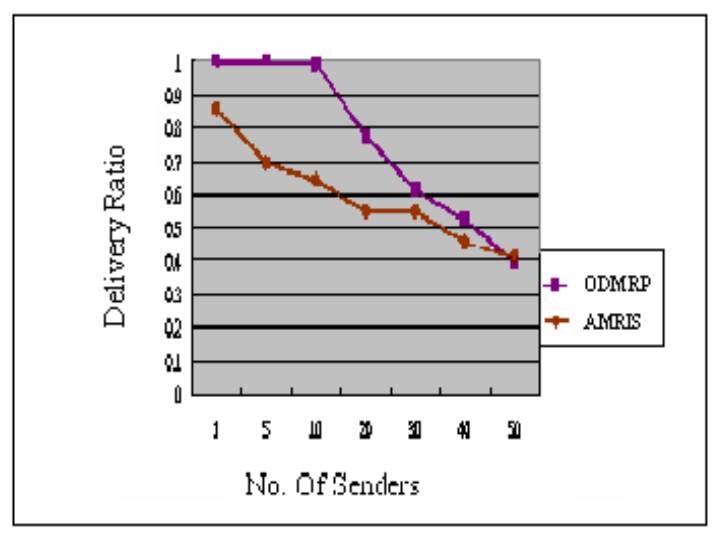

Fig 1. Delivery Ratio vs. No. of Senders (ODMRP and AMRIS) 
A comparison was made with ODMRP, AMRIS and MAODV. ODMRP uses a mesh structure to provide an efficient delivery ratio. AMRIS uses the traditional shared- based structure to construct a delivery tree and it needs a rendezvous point.

Various performance metrics were considered for 1 to 50 senders among a network of 50 nodes. The number of receivers was set to 5 . The simulation results are shown in Figure 1. Because each sender of ODMRP floods control messages into the entire network periodically, the packet collision probability becomes higher when the number of senders increases. The senders in the AMRIS protocol must forward data packets to a rendezvous point; the rendezvous point is very busy when many senders are sending data. This situation may also increase the packet collision probability.

As a starting set of simulations we have varied the number of senders to evaluate the protocol scalability based on the number of multicast source nodes and the traffic load. We inferred from the fig-2 that ODMRP is over 33\% more effective than MAODV in data delivery ratio as the number of senders incremented from $0-20$. We have also observed that all three protocols have not performed well if the number of senders increased above 20 .

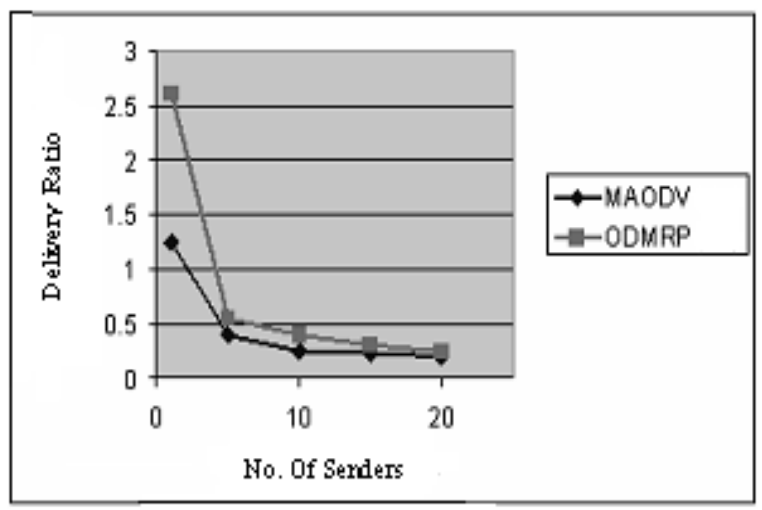

Fig 2. Delivery Ratio vs. No. of Senders (ODMRP and MAODV)

\subsection{Effect of Multicast Group Size}

ODMRP performance is not affected to that extent by the increase in the number of multicast members. AMRIS also shows improvement with the member size growth but they are less dramatic because redundant routes are not established in AMRIS. This is shown in fig. 3 where we see the comparison between ODMRP and AMRIS. With respect to the increase in the group member size MAODV is doing well than ODMRP, which can be attributed because of the collision that occur due to the frequent broadcast through the network. This is depicted in fig. 4 which shows the comparison between ODMRP and MAODV.

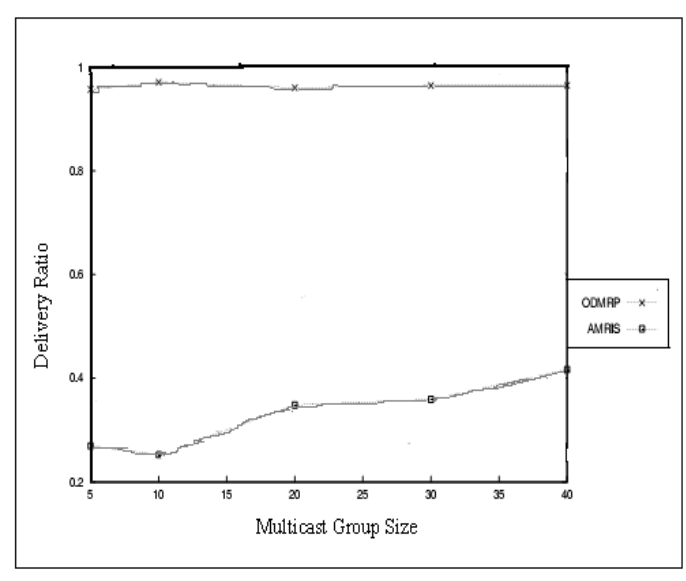

Fig 3. Delivery Ratio vs. Multicast Group Size (ODMRP and AMRIS)

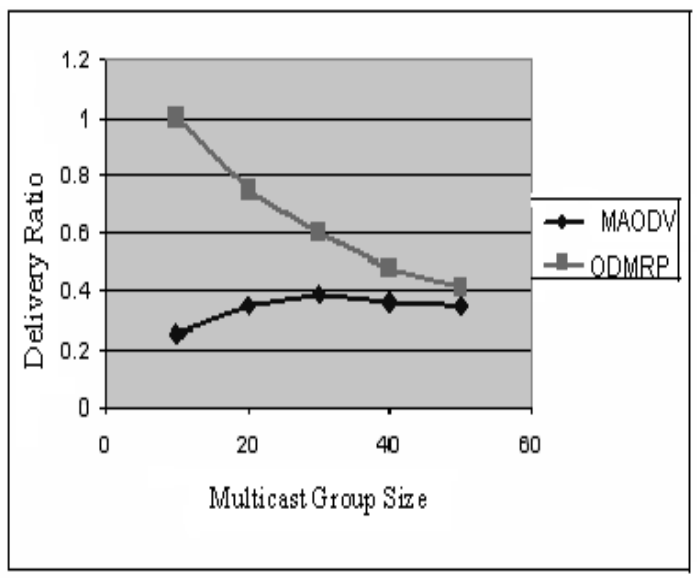

Fig 4. Delivery Ratio vs. Multicast Group Size (ODMRP and MAODV)

\section{CONCLUSION}

A general conclusion is that, in a mobile scenario, mesh-based protocols out performed tree-based protocols. The availability of alternate routes provided robustness to mobility. AMRIS was effective in a light traffic environment with no mobility, but its performance was susceptible to traffic load and mobility. ODMRP was very effective and efficient in most of our simulation scenarios. However, the protocol showed a trend of rapidly increasing overhead as the number of senders increased. The rudimentary area for improving the MAODV is the fragileness of the bi-directional shared tree which causing the poor delivery ratio. We experimented with scenarios which we thought were the most representation of ad hoc wireless network applications. However, we did not cover every possible situation. While the results of this paper can provide guidelines, the final selection of a multicast protocol should take into account other considerations which cannot be valuated via simulation alone. 


\section{REFERENCES}

[1] S.-J. Lee, M. Gerla, and C.-C. Chiang, "On-Demand Multicast Routing Protocol,' In Proceedings of IEEE WCNC'99, New Orleans, LA, Sep. 1999, pp. 1298-1304.

[2] S.-J. Lee, W. Su, and M. Gerla, "Ad hoc Wireless Multicast with Mobility Prediction," In Proceedings of IEEE ICCCN'99, Boston, MA, Oct. 1999, pp. 4-9.

[3] S.-J. Lee, W. Su, and M. Gerla, "On-Demand Multicast Routing Protocol (ODMRP) for Ad Hoc Networks," InternetDraft, draft-ietf-manet-odmrp-01.txt, Jun. 1999.
[4] C.W. Wu, Y.C. Tay, and C.-K. Toh, "Ad hoc Multicast Routing protocol utilizing Increasing id-numberS (AMRIS) Functional Specification," Internet-Draft, draft-ietf-manet-amrisspec-00.txt, Nov. 1998.

[5] Carlos de Morais Cordeiro, Hrishikesh Gossain, and Dharma P. Agrawal, University of Cincinnati, "Multicast over Wireless Mobile Ad Hoc Networks: Present and Future Directions".

[6] Elizabeth M. Royer and Charles E. Perkins. Multicast Operation of the Ad-hoc On-Demand Distance Vector Routing Protocol. In Proceedings of the Fifth Annual ACM/IEEE International Conference on Mobile Computing and Networking, Mobicom '99, pages 207-218, August 1999. 